\title{
INTENSIDAD DE LA LUZ EMITIDA POR LÁMPARAS DE FOTOCURADO EN LOS CONSULTORIOS ODONTOLÓGICOS DE BUCARAMANGA Y SU ÁREA METROPOLITANA
}

\author{
${ }^{1}$ Jenny Johana Matallana Jerez, ${ }^{1}$ Ángela Helena Ortiz Caballero, ${ }^{1}$ María Fernanda Rincón Capacho, ${ }^{1}$ Andrea Carolina Sánchez Carvajal, \\ ${ }^{2}$ Gloria Cristina Aránzazu Moya, ${ }^{3}$ Sonia Constanza Concha Sánchez. \\ ${ }^{1}$ Estudiante X semestre F de Odontología U. Santo Tomás. ${ }^{2}$ Odontóloga U. Santo Tomás, Especialista en Patología Oral y Medios Diagnósticos \\ U. El Bosque, Docente U. Santo Tomás. ${ }^{3}$ Odontóloga U. Santo Tomás, Especialista en Educación y Comunicación para la Salud, \\ MSc Epidemiología U. Industrial de Santander, Docente U. Santo Tomás.
}

Autor responsable de correspondencia: Andrea Carolina Sánchez C.

Correo electrónico: andreitasanchez88@hotmail.com

\begin{abstract}
RESUMEN
Objetivo: Determinar la intensidad de la luz de las lámparas de fotocurado empleadas en los consultorios odontológicos de Bucaramanga y su área metropolitana, y evaluar el conocimiento de los odontólogos acerca del tema.

Materiales y métodos: Se realizó un estudio observacional descriptivo de corte transversal a un universo de 283 y una muestra de 110 centros odontológicos, las variables incluidas en este estudio fueron las sociodemográficas (municipio, estrato socioeconómico), acerca de la lámpara de fotocurado (tipo de luz, marca, mantenimiento, personal que realiza el mantenimiento), acerca de la desinfección de la lámpara (sustancia utilizada y la frecuencia) y, variables de conocimiento del profesional acerca de la unidad de curado; se realizó el cálculo de medidas de resumen y para el análisis bivariado se aplicaron la prueba de Test Exacto de Fisher y ANOVA.

Resultados: Se encontraron asociaciones estadísticamente significativas de la intensidad de luz como nivel adecuado con las variables tipo de luz halógena con $59 \%(\mathrm{p}<0.001), 65.4 \%$ de las marcas de las lámparas sin referencia $(\mathrm{p}=0.013)$, el técnico era la persona que realizaba el mantenimiento en el 61.8\% ( $\mathrm{p}=0.033)$, el alcohol fue la sustancia utilizada para desinfectar en un 46.7\% $(\mathrm{p}=0.0117)$, el 61\% de los odontólogos tenía un conocimiento regular $(\mathrm{p}=0.012)$, entre otros.

Conclusión: El 21.9\% de las lámparas no tiene una intensidad de luz adecuada para polimerizar los materiales. [Matallana JJ, Ortiz AH, Rincón MF, Sánchez AC, Aránzazu GC, Concha SC. Intensidad de la luz emitida por lámparas de fotocurado en los consultorios odontológicos de Bucaramanga y su área metropolitana. Ustasalud 2010; 9: 41 - 49]
\end{abstract}

Palabras clave: Consultorios odontológicos, Lámpara de fotocurado, Intensidad de luz, Radiómetro.

\section{INTENSITY OF LIGHT-CURING UNITS USED IN PRIVATE PRACTICE IN BUCARAMANGA AND ITS METROPOLITAN AREA}

\begin{abstract}
Objective: To determine light intensity of light polimerization units used in the dental offices in Bucaramanga and its metropolitan area, and to evaluate the dentists' knowledge in this subject.

Methods: A descriptive cross section observational study was done. The universe was constituted by 283 and a sample of 110 dental offices was evaluated. The variables included were sociodemographical (municipality, socioeconomic status), about the light curing lamps (type of light, brand, maintenance, personnel performing the maintenance), about the lamp disinfestations (the substance that was used and frequency), and professional's knowledge about the polimerization unit. Means, modes, medians and standard deviation were determined for all data. Bivariate analyses were performed by Fisher Exact Test and ANOVA.

Results: There were found statistically significant differences of the intensity of light with the variables of type of light with 59\% $(\mathrm{p}<0.001)$, brand of the lamp without commercial reference with $65.4 \%(\mathrm{p}=0.013)$, personnel performing maintenance, the technician with $61.8 \%(p=0.033)$, the substance used to disinfect, alcohol with $46.7 \%(p=0.0117)$, variables such as knowledge of the dentist $61 \%$ regular knowledge $(\mathrm{p}=0.012)$.

Conclusion: It was determined that $21.9 \%$ of the lamps didn't have an appropriate intensity of light for curing the materials.
\end{abstract}

Key words: Dental clinics, Light curing lamp, Light intensity, Radiometer.

Recibido para publicación: 13 de noviembre de 2009. Aceptado para publicación: 10 de marzo de 2010 . 


\section{INTRODUCCIÓN}

La lámpara de fotocurado es un elemento importante en la práctica odontológica para la fotopolimerización de algunos materiales. Una intensidad de luz inadecuada puede producir alteraciones en las propiedades mecánicas del material restaurador, profundidad de curado, stress de contracción de la resina, GAP, microfiltración, sensibilidad postoperatoria, inflamación pulpar y caries secundaria, problemas que deben ser evitadas en la práctica odontológica. ${ }^{1-3}$

El desarrollo tecnológico ha sido realmente importante en los últimos años, lo que ha generado que el odontólogo tenga que tomar decisiones con respecto a estos avances científicos, evento que lleva implícito el conocimiento de las bases técnico-científicas que sostienen este avance y los requisitos que deben reunir estas unidades de polimerizado, así como las investigaciones que con esta tecnología se han llevado a cabo, para seleccionar de manera adecuada la unidad de polimerizado más conveniente para la práctica clínica. ${ }^{4}$

En Bucaramanga y su área metropolitana no se han realizado estudios donde se mida la intensidad de la luz existente en la lámpara de fotocurado, por tal razón el interrogante que da origen a la presente investigación se formula así: ¿Es adecuada la intensidad de la luz de las lámparas de fotocurado que se utilizan en los consultorios odontológicos del área metropolitana de Bucaramanga? ¿Los resultados relacionados con la intensidad de la luz serán similares a los observados en estudios reportados?

La pregunta formulada permite establecer como objetivo general, determinar la intensidad de la luz de las lámparas de fotocurado empleadas en los consultorios odontológicos de Bucaramanga y su área metropolitana durante el segundo semestre del 2008 y evaluar el conocimiento de los odontólogos acerca del tema.

\section{MATERIALES Y MÉTODOS}

Se realizó un estudio observacional descriptivo de corte transversal. El universo estuvo conformado por 283 centros odontológicos de Bucaramanga y su área metropolitana durante el segundo semestre del 2008. La muestra la integraron 110 centros odontológicos, el tipo de muestreo utilizado fue probabilístico, aleatorio simple. Se consideraron como criterios de inclusión consultorios odontológicos ubicados en Girón, Piedecuesta, Bucaramanga y Floridablanca, con lámparas de fotocurado LED, Halógenas, Arco de plasma y Láser, y los odontólogos que aceptaron voluntariamente participar en la investigación. Como criterios de exclusión se definieron las lámparas de fotocurado utilizadas exclusivamente para blanqueamiento dental.

\section{Se realizaron dos formatos:}

1. El primero incluía 14 preguntas: las dos primeras se relacionaban con variables sociodemográficas como municipio y estrato socioeconómico. Las siguientes cuatro preguntas hacían referencia a conceptos básicos generales sobre la luz de fotocurado tales como tipo de luz, marca de la lámpara, intensidad de la luz reportada por el radiómetro e intensidad de la luz como nivel adecuado. Las siete preguntas siguientes hacían referencia a la lámpara como años de uso reportados por el odontólogo, uso diario de la lámpara, mantenimiento de ésta, personal que realiza el mantenimiento, desinfección de la lámpara, sustancia y frecuencia con la que la realiza, la última pregunta correspondía al conocimiento del odontólogo evaluado por medio de una pregunta de autodiligenciamiento.

2. El segundo evaluó 13 preguntas sobre la unidad de medida de las lámparas de fotocurado, elemento con el cual se mide esta intensidad, si una mala intensidad de la luz de la lámpara de fotocurado podría producir: alteración a nivel ocular, sensibilidad dental, interfase diente material restaurador, caries secundaria, inflamación pulpar, sobrecalentamiento pulpar y muerte celular; revisión de la intensidad de luz de la lámpara a consideración del odontólogo, clasificación de la intensidad de luz que registró la lámpara, máximo espesor de resina y tiempo de curado por cada capa de resina empleado por el odontólogo.

Se efectuó una prueba piloto en la que se verificó la comprensión de las preguntas, los aspectos relacionados con la logística y el manejo de los datos. A partir de ésta se efectuaron los correctivos necesarios.

Posteriormente, se registraron los centros odontológicos de acuerdo a la guía telefónica de Telebucaramanga 2008-2009 para proceder a la recolección de la muestra. Se coordinó la aplicación de la encuesta a los consultorios seleccionados en los horarios de atención establecidos para donde se aplicaron los dos cuestionarios y la respectiva medición de la intensidad. 
Una vez recolectados los datos se procedió a la sistematizacion en Excel por duplicado, ${ }^{5}$ se transportó la base de datos a EPI- INFO 6.04 y mediante la rutina Validate se verificó la calidad de la digitación y se exportó al paquete estadístico STATA 9.0 para su correspondiente procesamiento y análisis. ${ }^{6}$

El plan de análisis univariado consistió en el cálculo de medidas de resumen según la naturaleza de las variables; para cualitativas se calculó proporción y para las cuantitativas medidas de tendencia central como media, mediana y para las de dispersión desviación estándar y rango.

En el análisis bivariado se consideró como variable de salida intensidad de luz como nivel adecuado la que se analizó con las otras variables del estudio; para las variables cualitativas se aplicó Test Exacto de Fisher y para las cuantitativas análisis de varianza de una sola vía (ANOVA).

Este trabajo se acogió a la normatividad establecida en la resolución 008430 de 1993 según el artículo 11 , que considera un estudio sin riesgo ya que se realizaron entrevistas, cuestionarios, medición de un aparato que no involucró aspectos sensitivos de la conducta del ser humano. ${ }^{7}$

\section{RESULTADOS}

\section{Análisis univariado}

\section{Aspectos sociodemográficos}

Se evaluaron las lámparas de fotocurado de 110 consultorios odontológicos de Bucaramanga y su área metropolitana; el 85.5\% (94) correspondían a Bucaramanga, el $52.7 \%$ (58) pertenecían al estrado 6, mientras que en el estrato 2 sólo se encontró una lámpara (0.9\%) (Tabla 1$)$.

\section{Variables acerca de la lámpara de fotocurado}

El tipo de la luz más utilizado en los consultorios odontológicos fue la halógena 59\% (65). De los items de respuesta de la marca de lámpara de fotocurado la más utilizada fue la $3 \mathrm{M} \mathrm{ESPE}^{\circledR}$ con el $14.6 \%$ (16), seguida del $13.6 \%$ (15) de la Optilux ${ }^{\circledR}$. Sin embargo, el 65.4\% (72) pertenecían a otra marca diferente de las opciones dadas, que no se pudo identificar. En cuanto al mantenimiento de la lámpara, el $41.8 \%$ (46) rara vez lo realiza (mayor 6 meses), (Tabla 2). Al medir la intensidad de luz reportada por el radiómetro, el promedio fue de $600 \pm 627$ con rango de intensidad entre 42 $\mathrm{mw} / \mathrm{cm}^{2}$ y $1775 \mathrm{mw} / \mathrm{cm}^{2}$.

Tabla 1. Descripción de las variables sociodemográficas analizadas en forma global y según la intensidad de la luz.

\begin{tabular}{|c|c|c|c|c|c|c|c|}
\hline \multirow[b]{2}{*}{ VARIABLE } & \multirow{2}{*}{$\begin{array}{l}\text { GLOBAL } \\
\text { Frec (\%) }\end{array}$} & \multicolumn{5}{|c|}{ CLASIFICACIÓN DE LA INTENSIDAD DE LA LUZ } & \multirow[b]{2}{*}{$P$} \\
\hline & & $\begin{array}{l}\text { No Aptas } \\
\text { Frec. (\%) }\end{array}$ & $\begin{array}{c}\text { I. Baja } \\
\text { Frec. (\%) }\end{array}$ & $\begin{array}{l}\text { I. Media } \\
\text { Frec. (\%) }\end{array}$ & $\begin{array}{l}\text { I. Alta } \\
\text { Frec. (\%) }\end{array}$ & $\begin{array}{l}\text { I. Super Alta } \\
\text { Frec. (\%) }\end{array}$ & \\
\hline Global & $110(100)$ & $25(22.7)$ & $20(18.2)$ & $23(20.9)$ & $26(23.6)$ & $16(14.6)$ & \\
\hline Municipio & & & & & & & $0,520^{*}$ \\
\hline Bucaramanga & $94(85.5)$ & $20(21.2)$ & $15(16)$ & $20(21.2)$ & $25(26.6)$ & $14(14.9)$ & \\
\hline Floridablanca & $14(12.7)$ & $4(28.6)$ & $4(28.6)$ & $3(21.4)$ & $1(7.1)$ & $2(14.3)$ & \\
\hline Piedecuesta & $2(1.8)$ & $1(50)$ & $1(50)$ & --- & --- & --- & \\
\hline Estrato & & & & & & & $0,390^{*}$ \\
\hline Estrato 1 & $2(1.8)$ & $1(50)$ & $1(50)$ & --- & --- & --- & \\
\hline Estrato 2 & $1(0.9)$ & --- & --- & --- & --- & --- & \\
\hline Estrato 3 & $9(8.1)$ & $4(44.4)$ & $3(33.3)$ & $1(11.1)$ & $1(11.1)$ & --- & \\
\hline Estrato 4 & 15 (13.6) & $4(26.7)$ & $1(6.7)$ & $4(26.7)$ & $4(26.7)$ & $2(13.3)$ & \\
\hline Estrato 5 & $25(22.7)$ & $4(16)$ & $2(8)$ & $9(36)$ & $6(24)$ & $4(16)$ & \\
\hline Estrato 6 & 58 (52.7) & $12(20.7)$ & $13(22.4)$ & $9(15.5)$ & $15(25.8)$ & $9(15.5)$ & \\
\hline
\end{tabular}

* Test Exacto de Fisher. 


\section{Variable de desinfección}

En el $4.5 \%$ (5) de los consultorios no desinfectan la guía de luz de la lámpara, en los que sí lo realizan utilizan el alcohol $46.7 \%$ (50) como sustancia desinfectante con una frecuencia del $67.3 \%(72)$ realizada entre paciente y paciente (Tabla 3).

Tabla 2. Descripción de las variables acerca de la lámpara de fotocurado analizadas en forma global y según la intensidad de la luz.

\begin{tabular}{|c|c|c|c|c|c|c|c|}
\hline \multirow[b]{2}{*}{ VARIABLE } & \multirow[b]{2}{*}{$\begin{array}{l}\text { GLOBAL } \\
\text { Frec (\%) }\end{array}$} & \multicolumn{5}{|c|}{ CLASIFICACIÓN DE LA INTENSIDAD DE LA LUZ } & \multirow[b]{2}{*}{$P$} \\
\hline & & $\begin{array}{l}\text { No Aptas } \\
\text { Frec. (\%) }\end{array}$ & $\begin{array}{c}\text { I. Baja } \\
\text { Frec. (\%) }\end{array}$ & $\begin{array}{l}\text { I. Media } \\
\text { Frec. (\%) }\end{array}$ & $\begin{array}{c}\text { I. Alta } \\
\text { Frec. (\%) }\end{array}$ & $\begin{array}{l}\text { I. Super Alta } \\
\text { Frec. (\%) }\end{array}$ & \\
\hline Global & $110(100)$ & $25(22.7)$ & $20(18.2)$ & $23(20.9)$ & $26(23.6)$ & $16(14.6)$ & \\
\hline Tipo de luz & & & & & & & $<0,001^{*} 1$ \\
\hline LED & $45(41)$ & $5(11.1)$ & --- & $8(17.8)$ & $17(37.8)$ & 15 (33.4) & \\
\hline Halógena & $65(59)$ & $20(30.8)$ & $20(30.8)$ & $15(23.1)$ & $9(13.9)$ & $1(1.5)$ & \\
\hline Marca & & & & & & & $0,013^{*}$ \\
\hline Dentsply & $6(5.5)$ & --- & $2(33.3)$ & $4(66.7)$ & --- & --- & \\
\hline Optilux & 15 (13.6) & $4(26.7)$ & $7(46.7)$ & $1(6.7)$ & $2(13.3)$ & $1(6.7)$ & \\
\hline 3M Espe & 16 (14.6) & $3(18.8)$ & $2(12.5)$ & $1(6.3)$ & $5(31.3)$ & $5(31.3)$ & \\
\hline VIP & $1(0.91)$ & -- & -- & $1(100)$ & --- & --- & \\
\hline Otras & $72(65.4)$ & $18(25)$ & 9 (12.5) & $16(22.2)$ & $19(26.4)$ & 10 (13.9) & \\
\hline Mantenimiento & & & & & & & $0,257^{*}$ \\
\hline $\begin{array}{l}\text { Frecuentemente } \\
\text { (cada mes) }\end{array}$ & $4(3.6)$ & --- & -- & -- & $2(50)$ & $2(50)$ & \\
\hline $\begin{array}{l}\text { Ocasionalmente } \\
\text { (> } 1-6 \text { meses) }\end{array}$ & $28(25.5)$ & $7(25)$ & $4(14.3)$ & $5(17.9)$ & $8(28.6)$ & $4(14.3)$ & \\
\hline $\begin{array}{l}\text { Rara vez } \\
\text { (> a } 6 \text { meses) }\end{array}$ & $46(41.8)$ & $11(23.9)$ & $13(28.3)$ & 9 (19.6) & $10(21.7)$ & $3(6.5)$ & \\
\hline Nunca & $32(29.1)$ & $7(21.9)$ & $3(9.4)$ & 7 (21.9) & $8(25)$ & $7(21.9)$ & \\
\hline Personal & & & & & & & $0,033^{*}$ \\
\hline No se realiza & $33(30)$ & $7(21.2)$ & $4(12.1)$ & $7(21.2)$ & $8(24.2)$ & $7(21.2$ & \\
\hline $\begin{array}{l}\text { Personal del } \\
\text { consultorio }\end{array}$ & $9(8.2)$ & $3(33.3)$ & $6(66.7)$ & --- & --- & -- & \\
\hline Técnico & $68(61.8)$ & $15(22.1)$ & 10 (14.7) & $16(23.5)$ & $18(26.5)$ & $9(13.2)$ & \\
\hline
\end{tabular}

Tabla 3. Descripción de las variables de desinfección analizadas en forma global y según la intensidad de la luz.

\begin{tabular}{|c|c|c|c|c|c|c|c|}
\hline \multirow[b]{2}{*}{ VARIABLE } & \multirow[b]{2}{*}{$\begin{array}{l}\text { GLOBAL } \\
\text { Frec (\%) }\end{array}$} & \multicolumn{5}{|c|}{ CLASIFICACIÓN DE LA INTENSIDAD DE LA LUZ } & \multirow[b]{2}{*}{$P$} \\
\hline & & $\begin{array}{l}\text { No Aptas } \\
\text { Frec. (\%) }\end{array}$ & $\begin{array}{c}\text { I. Baja } \\
\text { Frec. (\%) }\end{array}$ & $\begin{array}{l}\text { I. Media } \\
\text { Frec. (\%) }\end{array}$ & $\begin{array}{c}\text { I. Alta } \\
\text { Frec. (\%) }\end{array}$ & $\begin{array}{l}\text { I. Super Alta } \\
\text { Frec. (\%) }\end{array}$ & \\
\hline Global & $110(100)$ & $25(22.7)$ & $20(18.2)$ & 23 (20.9) & 26 (23.6) & 16 (14.6) & \\
\hline Desinfencción & & & & & & & $0,345^{*}$ \\
\hline $\mathrm{Si}$ & 105 (95.5) & $22(20.9)$ & 19 (18.1) & 23 (21.9) & $25(23.8)$ & $16(15.2)$ & \\
\hline No & $5(4.5)$ & $3(60)$ & $1(20)$ & --- & $1(20)$ & --- & \\
\hline Sustancia & & & & & & & $0,0117^{*} 1$ \\
\hline Hipoclorito & $12(11.2)$ & $4(33.3)$ & $5(41)$. & $3(25)$ & --- & -- & \\
\hline Alcohol & $50(46.7)$ & $9(18)$ & $9(18)$ & $10(20)$ & $14(28)$ & $8(16)$ & \\
\hline Glutaraldehído & $41(38.3)$ & $10(24.4)$ & $5(12.2)$ & $10(24.4)$ & $10(24.4)$ & $6(14.6)$ & \\
\hline Otras & $4(3.7)$ & -- & --- & --- & $2(50)$ & $2(50)$ & \\
\hline Frecuencia & & & & & & & $0,494^{*}$ \\
\hline Entre pacientes & $72(67.3)$ & $13(18.1)$ & $11(15.3)$ & $17(23.6)$ & $18(25)$ & $13(18.1)$ & \\
\hline Final de la jornada & $23(21.5)$ & $5(21.7)$ & $6(26.1)$ & $4(17.4)$ & $5(21.7)$ & $3(13)$ & \\
\hline Semanalmente & $7(6.5)$ & $4(57.1)$ & --- & $2(28.6)$ & $1(14.3)$ & --- & \\
\hline Otros & $5(4.7)$ & $1(20)$ & $2(40)$ & --- & $2(40)$ & -- & \\
\hline
\end{tabular}

* Test Exacto de Fisher - 1 Estadísticamente significativo. 


\section{Conocimiento del odontólogo}

El 61\% (64) de los odontólogos encuestados registraron un conocimiento regular, sólo el 1\% (1) obtuvo un conocimiento bueno sobre lámparas de fotocurado y los daños que causa una intensidad de luz inadecuada (Tabla 4).

En cuanto a las preguntas que se emplearon para evaluar el conocimiento del odontólogo se encontró que el $27.6 \%$ (29) no conocen cuál es la unidad de medida de la intensidad de la luz de la lámpara de fotocurado. Con respecto a los daños que ocasiona una mala intensidad de luz, el 50.5\% (54) de los odontólogos respondieron que no ocasiona daño a nivel ocular. Al tener en cuenta las otras variables para evaluar el conocimiento del odontólogo como fueron sensibilidad dental el 6.7\% (7), interfase diente-restauración 7.6\% (8), caries secundaria $9.5 \%$ (10), inflamación pulpar 13.3\% (14), sobrecalentamiento pulpar $4.8 \%$ (5) y muerte celular el $15.2 \%$ (16) no conocen si causa o no algún daño (Tabla 5).

El 66.7\% (70) de los odontólogos consideraron que la intensidad de luz de la lámpara se debe revisar cada seis meses, sólo el 3.8\% (4) consideró que debe revisarse diariamente (Tabla 4).

\section{Análisis bivariado}

\section{Aspectos sociodemográficos y clasificación de la intensidad de luz}

$\mathrm{Al}$ analizar la intensidad de luz de los consultorios odontológicos de acuerdo a las variables sociodemográficas no se encontraron asociaciones estadísticamente significativas para las variables municipio y estrato. Para el estrato $1 \mathrm{y}$ el municipio de Piedecuesta se encontraron dos lámparas de las cuales el 50\% (1) no eran aptas para polimerizar y el otro $50 \%$ (1) tuvo una intensidad de luz baja. En el estrato 3, el $44.4 \%$ (4) no eran aptas para polimerizar el material restaurador (Tabla 1).

Tabla 4. Descripción de las variables conocimiento en forma global y según la intensidad de la luz.

\begin{tabular}{|c|c|c|c|c|c|c|c|}
\hline \multirow[b]{2}{*}{ VARIABLE } & \multirow[b]{2}{*}{$\begin{array}{l}\text { GLOBAL } \\
\text { Frec (\%) }\end{array}$} & \multicolumn{5}{|c|}{ CLASIFICACIÓN DE LA INTENSIDAD DE LA LUZ } & \multirow[b]{2}{*}{$\mathrm{P}$} \\
\hline & & $\begin{array}{l}\text { No Aptas } \\
\text { Frec. (\%) }\end{array}$ & $\begin{array}{l}\text { I. Baja } \\
\text { Frec. (\%) }\end{array}$ & $\begin{array}{l}\text { I. Media } \\
\text { Frec. (\%) }\end{array}$ & $\begin{array}{l}\text { I. Alta } \\
\text { Frec. (\%) }\end{array}$ & $\begin{array}{l}\text { I. Super } \\
\text { Alta } \\
\text { Frec. (\%) }\end{array}$ & \\
\hline Global & $105(100)$ & $23(21.9)$ & $18(17.1)$ & $23(21.9)$ & $25(23.8)$ & $16(15.2)$ & \\
\hline Conocimiento & & & & & & & $0,012 * 1$ \\
\hline Malo & $40(38.1)$ & $12(30)$ & $12(30)$ & $4(10)$ & 7 (17.5) & $5(12.5)$ & \\
\hline Regular & $64(61)$ & $11(17.2)$ & $6(9.4)$ & $18(28.1)$ & $18(28.1)$ & $11(17.2)$ & \\
\hline Bueno & $1(0.1)$ & --- & --- & $1(100)$ & -- & --- & \\
\hline Unidad de medida & & & & & & & 0,687 \\
\hline $\mathrm{cm} / \mathrm{mw}$ & $3(2.9)$ & $1(33.3)$ & $1(33.3)$ & --- & $1(33.3)$ & --- & \\
\hline $\mathrm{mw}$ & $25(23.8)$ & $7(28)$ & $4(16)$ & $4(16)$ & $6(24)$ & $4(16)$ & \\
\hline $\mathrm{mw} / \mathrm{cm}^{2}$ & $48(45.7)$ & $7(14.6)$ & $7(14.6)$ & $15(31.3)$ & $10(20.8)$ & $9(18.8)$ & \\
\hline No conoce & $29(27.6)$ & $8(26.7)$ & $6(20.7)$ & $4(13.8)$ & $8(27.6)$ & $3(10.3)$ & \\
\hline $\begin{array}{l}\text { Frecuencia revisión de } \\
\text { intensidad de luz }\end{array}$ & & & & & & & $0,101^{*}$ \\
\hline Diariamente & $4(3.6)$ & $1(25)$ & --- & --- & $1(25)$ & --- & \\
\hline Semanal & $5(4.8)$ & $3(60)$ & --- & $1(20)$ & $1(20)$ & --- & \\
\hline Cada mes & $24(22.9)$ & $2(8.3)$ & $3(12.5)$ & $10(41.7)$ & $6(25)$ & $3(12.5)$ & \\
\hline Cada seis meses & $70(66.7)$ & $17(24.3)$ & $15(21.4)$ & $12(17.1)$ & $15(21.4)$ & $11(15.7)$ & \\
\hline Otros & $2(1.9)$ & --- & --- & --- & $2(100)$ & --- & \\
\hline Espesor de la resina & & & & & & & $0,799^{*}$ \\
\hline $0.5 \mathrm{~mm}$ & $30(28.6)$ & $7(23.3)$ & $8(26.7)$ & $4(13.3)$ & $7(23.3)$ & $4(13.3)$ & \\
\hline $3 \mathrm{~mm}$ & $7(6.7)$ & $2(28.6)$ & $1(14.3)$ & $2(28.6)$ & $1(14.3)$ & $1(14.3)$ & \\
\hline $2 \mathrm{~mm}$ & 65 (61.9) & $14(21.5)$ & $8(12.3)$ & $16(24.6)$ & $17(26.1)$ & $10(15.4)$ & \\
\hline No conoce & $3(2.9)$ & -- & $1(33.3)$ & $1(33.3)$ & --- & $1(33.3)$ & \\
\hline
\end{tabular}

* Test Exacto de Fisher - 1 Estadísticamente significativo. 


\section{Variables relacionadas con la lámpara de foto- curado y clasificacion de la intensidad de luz}

$\mathrm{Al}$ comparar cada una de las variables relacionadas con la lámpara de fotocurado se encontraron asociaciones estadísticamente significativas para tipo de luz ( $\mathrm{P}<0,001)$; para marca de la lámpara $(P=0,013)$ y para personal que realiza el mantenimiento $(P=0,033)$.

Para el tipo de luz que emiten las lámparas de fotocurado se encontró que el $30.8 \%$ (20) de las halógenas y el $11.1 \%$ (5) de las LED no eran aptas para polimerizar el material restaurador, mientras que el $33.4 \%$ (15) de las LED y el $1.5 \%$ (1) de las halógenas se encontraron con una intensidad super alta. Al evaluar las lámparas no aptas para polimerizar, Optilux $26.7 \%$ (4) fue la marca con mayor porcentaje, seguida del $25 \%$ (18) de otras marcas comerciales, de las cuales no se logró identificar la marca.

En cuanto al mantenimiento que se realiza a la lámpara de fotocurado, el 50\% (2) que lo realiza frecuentemente tiene su lámpara en intensidad alta y el otro $50 \%$ (2) en intensidad super alta. Para los consultorios que nunca realizan mantenimiento se encontró para lámparas no aptas, intensidad media y super alta, el mismo porcentaje $21.2 \%$ (7) (Tabla 2).

\section{Desinfección y clasificación de intensidad de luz}

Al analizar las variables de desinfección sólo se observó asociación estadísticamente significativa en la sustancia utilizada para desinfectar $(P=0,0117)$. Al utilizar el hipoclorito como sustancia de desinfección, las lámparas se clasificaron como no aptas $33.3 \%$ (4) y de intensidad baja $41.7 \%$ (5), esta sustancia no fue utilizada en lámparas de intensidad alta y super alta (Tabla 3 ).

\section{Conocimiento del odontólogo y clasificación de la intensidad de luz}

Se encontraron asociaciones estadísticamente significativas en variables como conocimiento del odontólogo $(\mathrm{P}=0,012)$, sobrecalentamiento pulpar $(\mathrm{P}=$ $0,050)$ y muerte celular $(P=0.050)$ (Tabla 4 y 5$)$. El mal conocimiento del odontólogo se reflejó en lámparas no aptas para polimerizar, 30\% (12) y de intensidad baja, 30\% (12). Sólo un odontólogo obtuvo un buen conocimiento y su lámpara se clasificó en intensidad media, $100 \%$ (1) (Tabla 4).

\section{Frecuencia de uso de la lámpara y clasifica- ción de la intensidad de luz}

El mayor tiempo de años de uso lo obtuvieron las lámparas con intensidad baja (1.8 \pm 0.5 años), se- guido de las no aptas para polimerizar $(1.6 \pm 0.5$ años) y en menor tiempo de utilidad las lámparas con intensidad super alta (0.6 \pm 0.6 años) (Tabla 6).

\section{DISCUSIÓN}

Las lámparas de fotocurado aparecieron hace más de treinta años, éstas emitían una luz ultravioleta, según Radzi y colaboradores; ${ }^{8}$ su uso fue descontinuado porque requerian bastante tiempo de polimerización lo que causaba daños en la dentina y alteraban la microflora de la cavidad. Por tal motivo, surgieron nuevas lámparas con fuentes de luz de arco de plasma, láser, halógena y LED, estas dos últimas son las más utilizadas por los centros odontológicos de este estudio con un porcentaje de $59 \%$ y $41 \%$, respectivamente.

Estudios de similares características en los que se evaluó la intensidad de la luz mostraron que las lámparas de fotocurado no eran adecuadas para la fotopolimerización; en Envigado y Sabaneta (2002), el $18.4 \%$ de las lámparas eran inadecuadas para polimerizar, ${ }^{9}$ en Texas (1994) el 30\%, ${ }^{10}$ en Lancashire y norte de Manchester se registró que la intensidad de luz era inadecuado en el $28 \%$ de las lámparas evaluadas. ${ }^{11}$ En este estudio, el $22.7 \%$ registraron intensidades no aptas para polimerizar. Es necesario resaltar que en cada uno de los estudios, la clasificación de la intensidad y el número de lámparas revisadas eran diferentes.

La inadecuada intensidad de luz de las unidades de fotocurado puede producir alteraciones en las propiedades mecánicas del material restaurador, profundidad de curado y estrés de contracción de la resina. ${ }^{1}$ Según Säilynoja y colaboradores, desde el punto de vista clínico, el encogimiento por polimerización puede llevar a fallas adhesivas o cohesivas del material de restauración dado al estrés interno. ${ }^{12}$ Mowafy y colaboradores refieren que una de las propiedades biológicas de los materiales que podría ser alterada es la citotoxicidad posiblemente por la ingestión de monómeros u otros químicos que pueden tener lugar con incrementos relativamente mayores que no son bien polimerizados por las unidades de fotocurado. ${ }^{13}$

El encogimiento de polimerización puede resultar en GAP entre la cavidad y la resina, la formación de este contribuye a la microfiltración, permite el ingreso de fluidos como bacterias, moléculas, iones o aire en la interfase entre los materiales de restauración y la cavidad preparada, crea un microambiente dentro de la restauración, sensibilidad postoperatoria, inflamación pulpar y caries secundaria. ${ }^{2,3,12}$ Para evitar las alteraciones nombradas, se ha sugerido que es importante medir la intensidad de luz de 
la unidad de curado a través del radiómetro, como mínimo semanalmente; ${ }^{11,14,15}$ aunque existe una controversia sobre la periodicidad de la revisión de la intensidad de la luz, algunos manuales de casas comerciales como la Kerr y Dentsply sugieren que la intensidad debería revisarse diariamente. ${ }^{16,17}$ En este estudio sólo el 3.8\% de los odontólogos consi- dera que debe realizarse diariamente, el $66.7 \%$ creía que debería revisarse cada seis meses. Mitton y Wilson recomendaron que se deben registrar las lecturas tomadas de la intensidad de la luz, y si estas se encuentran por debajo de $300 \mathrm{mw} / \mathrm{cm}^{2}$ o si varían más o menos entre $50 \mathrm{mw} / \mathrm{cm}^{2}$ se requiere cambiar el bombillo o revisar la lámpara. ${ }^{11}$

Tabla 5. Descripción de las variables daños que ocasiona una mala intensidad de la luz de las lámparas según la clasificación de la intensidad.

\begin{tabular}{|c|c|c|c|c|c|c|c|}
\hline \multirow[b]{2}{*}{ VARIABLE } & \multirow[b]{2}{*}{$\begin{array}{l}\text { GLOBAL } \\
\text { Frec (\%) }\end{array}$} & \multicolumn{5}{|c|}{ CLASIFICACIÓN DE LA INTENSIDAD DE LA LUZ } & \multirow[b]{2}{*}{$\mathrm{P}$} \\
\hline & & $\begin{array}{l}\text { No Aptas } \\
\text { Frec. (\%) }\end{array}$ & $\begin{array}{c}\text { I. Baja } \\
\text { Frec. (\%) }\end{array}$ & $\begin{array}{l}\text { I. Media } \\
\text { Frec. (\%) }\end{array}$ & $\begin{array}{l}\text { I. Alta } \\
\text { Frec. (\%) }\end{array}$ & $\begin{array}{l}\text { I. Super } \\
\text { Alta } \\
\text { Frec. (\%) }\end{array}$ & \\
\hline Global & $105(100)$ & $23(21.9)$ & $18(17.1)$ & $23(21.9)$ & $25(23.8)$ & $16(15.2)$ & \\
\hline A nivel ocular & & & & & & & $0.520^{*}$ \\
\hline $\mathrm{Si}$ & 44 (41.9) & $8(18.2)$ & 7 (15.9) & $9(20.4)$ & $14(31.8)$ & $6(13.6)$ & \\
\hline No & $53(50.5)$ & $12(22.6)$ & $8(15.1)$ & $13(24.5)$ & $11(20.8)$ & $9(17)$ & \\
\hline No conoce & $8(7.6)$ & $3(37.5)$ & $3(37.5)$ & $1(12.5)$ & --- & $1(12.5)$ & \\
\hline Sensibilidad & & & & & & & $0,230^{*}$ \\
\hline Si & $78(74.3)$ & $12(21.8)$ & $13(16.7)$ & $14(1.9)$ & $21(26.9)$ & $13(16.7)$ & \\
\hline No & $20(19.1)$ & $3(15)$ & $5(25)$ & $5(25)$ & $4(20)$ & $3(15)$ & \\
\hline No conoce & $7(6.7)$ & $3(42.9)$ & --- & $4(57.1)$ & --- & --- & \\
\hline Interfase & & & & & & & $0,301^{*}$ \\
\hline $\mathrm{Si}$ & $84(80)$ & $17(20.2)$ & $12(14.3)$ & $21(25)$ & $19(22.6)$ & 15 (17.9) & \\
\hline No & $13(12.4)$ & $3(23.1)$ & $5(38.5)$ & $1(7.7)$ & $4(30.8)$ & -- & \\
\hline No conoce & $8(7.6)$ & $3(37.5)$ & $1(12.5)$ & $1(12.5)$ & $2(25)$ & $1(12.5)$ & \\
\hline Caries secundaria & & & & & & & $0,188^{*}$ \\
\hline $\mathrm{Si}$ & $60(57.1)$ & $13(21.7)$ & 7 (11.7) & $18(30)$ & $12(20)$ & $10(16.7)$ & \\
\hline No & $35(33.3)$ & $9(25.7)$ & $8(22.9)$ & $5(14.3)$ & $9(25.7)$ & $4(11.4)$ & \\
\hline No conoce & $10(9.5)$ & $1(10)$ & $3(30)$ & -- & $4(40)$ & $2(20)$ & \\
\hline Inflamación pulpar & & & & & & & $0.077^{*}$ \\
\hline $\mathrm{Si}$ & $64(61)$ & $11(17.2)$ & 10 (15.6) & $19(29.7)$ & $15(23.4)$ & $9(14.1)$ & \\
\hline No & $27(25.7)$ & $6(22.2)$ & $6(22.2)$ & $1(3.7)$ & 9 (33.3) & $5(18.5)$ & \\
\hline No conoce & $14(13.3)$ & $6(42.9)$ & $2(14.3)$ & $3(21.4)$ & $1(7.14)$ & $2(14.3)$ & \\
\hline Sobrecalentamiento & & & & & & & $0,050^{*} 1$ \\
\hline $\mathrm{Si}$ & $68(64.7)$ & $18(26.5)$ & $10(14.7)$ & $16(23.5)$ & $16(23.5)$ & $8(11.8)$ & \\
\hline No & $32(30.5)$ & $3(9.4)$ & 7 (21.9) & $5(15.6)$ & $9(28.1)$ & $8(25)$ & \\
\hline No conoce & $5(4.8)$ & $2(40)$ & $1(20)$ & $2(40)$ & --- & --- & \\
\hline \multicolumn{8}{|l|}{ Muerte celular } \\
\hline $\mathrm{Si}$ & $43(41)$ & $10(23.3)$ & $3(7)$ & $12(27.9)$ & 12 (27.9) & $6(14)$ & \\
\hline No & $46(43.8)$ & $7(15.2)$ & $10(21.7)$ & $10(21.7)$ & $9(19.6)$ & $10(21.7)$ & \\
\hline No conoce & $16(15.2)$ & $6(30.4)$ & $5(31.1)$ & $1(6.25)$ & $4(25)$ & --- & \\
\hline
\end{tabular}

* Test Exacto de Fisher - 1 Estadísticamente significativo. 
Tabla 6. Relaciona la frecuencia de uso de la lámpara de fotocurado según la intensidad de la luz.

\begin{tabular}{lcccccc}
\hline & \multicolumn{5}{c}{ CLASIFICACIÓN DE LA INTENSIDAD DE LA LUZ } & \multirow{2}{*}{ VARIABLE } \\
\cline { 2 - 6 } & No Aptas & I. Baja & I. Media & I. Alta & I. Super & Plta \\
& X D.E. & X \pm D.E & X \pm D.E & X \pm D.E & X \pm D.E & \\
\hline Años de uso & $1.6 \pm 0.5$ & $1.8 \pm 0.5$ & $1.4 \pm 0.8$ & $1.0 \pm 0.6$ & $0.6 \pm 0.6$ & $<0,0001^{* *}$ \\
Uso diario (horas) & $0.36 \pm 0.5$ & $0.3 \pm 0.6$ & $0.1 \pm 0.5$ & $0.4 \pm 0.7$ & $0.6 \pm 0.7$ & $0,2263^{* *}$ \\
\hline
\end{tabular}

** Test Anova.

Mowafy y colaboradores refieren que un filtro defectuoso no puede funcionar perfectamente, como resultado la longitud de onda de la luz producida no podría estar en el rango requerido. ${ }^{13}$ Además, éste afectará negativamente la intensidad de luz, por lo que es posible que aunque la intensidad de luz de una unidad sea adecuada, esta longitud de onda incorrecta afecte la lámpara de manera que no funcione eficazmente.

Este estudio demostró que en los consultorios de Bucaramanga y su área metropolitana no tenían radiómetro para medir la intensidad de la luz de sus lámparas de fotocurado, y en el momento de hacer el mantenimiento muchos de los técnicos tampoco poseían este aparato. Es de gran importancia que éste sea un elemento indispensable en la práctica clínica de los odontólogos para evitar las diferentes alteraciones que causa una mala intensidad de la luz. Sin embargo, Mitton y Wilson recomiendan que en ausencia del radiómetro, se haga una columna de $3 \mathrm{~mm}$ de material fotocurable sobre una superficie de trabajo, se fotocure desde arriba por 40 segundos, luego se verifique la dureza de la base de la columna. Si se puede retirar material parcialmente curado de la base de la columna se requiere mantenimiento de la lámpara. ${ }^{11}$

Las unidades de curado con más de 5 años de uso regular deben cambiarse, los resultados en este estudio arrojaron que el $44 \%$ de los consultorios odontológicos tienen la lámpara hace más de tres años y sólo el $1 \%$ la tiene hace más de 8 , aunque este es un rango muy grande debido a que no se sabe cuántos la tienen más de 5 años y con un uso regular que puedan ameritar su reposición.

En la guía de fotocurado realizada por Mitton y Wilson se recomienda que la punta de la guía de luz de la lámpara debe estar libre de materiales adhesivos, especialmente en el área que emite la luz, además debe ser esterilizada entre pacientes. ${ }^{11}$ En este estudio se evaluó la desinfección y no la esterilización, en los consultorios se utilizó alcohol como desifectante, en el 46,7\%. Para posteriores investigaciones, se recomienda evaluar estos factores, ya que influyen en la calidad de la intensidad de la luz. También es necesario tener en cuenta el grado de uso de la lámpara de fotocurado ya que en algunos consultorios no se usa tanto como en otros debido a la dedicación del odontólogo o al tipo de pacientes que se atienda.

A diferencia de los estudios anteriores, este estudio evaluó el conocimiento de los odontólogos que hicieron parte de la muestra. Los resultados arrojaron que los profesionales en esta área no tenían suficiente claridad sobre las preguntas realizadas, ya que el $61 \%$ obtuvieron un conocimiento regular y el $38 \%$ un conocimiento malo.

Los odontólogos necesitan acoger políticas para revisar periódicamente la eficacia de las lámparas de fotocurado de sus consultorios; deben verificarse lámparas, guías y filtros, y reemplazar por nuevos componentes cuando haya evidencia de deterioro. Las casas comerciales de lámparas de fotocurado deben considerar la organización de una campaña de conocimiento para probar, reparar y reemplazar las unidades defectuosas.

Por ende, es necesario que la academia brinde alternativas para que los profesionales estén actualizados en mantener su lámpara en óptimas condiciones, estas alternativas podrían ser formales (educación continua) y no formales (correos electrónicos o plegables).

\section{Conclusiones}

Se determinó que la intensidad de luz de las lámparas de fotocurado de los consultorios de $\mathrm{Bu}$ caramanga y su área metropolitana registran intensidades aceptables. Sin embargo, es de mayor impacto el porcentaje de lámparas no aptas para polimerizar $22.7 \%$ que genera alteraciones en la cavidad oral de los pacientes y propiedades del material restaurador.

El 38.1\% y el $61 \%$ de los odontólogos evidenciaron un conocimiento malo y regular respectiva- 
mente, por lo que es necesario que el profesional esté en constante actualización para reforzar y aclarar los conceptos obtenidos y así, mejore su práctica clínica.

Finalmente, los resultados obtenidos en este estudio demostraron que el profesional en odontología debe prestar especial atención en los servicios ofrecidos para conducir a un nivel superior de satisfacción del paciente.

\section{BIBLIOGRAFÍA}

1. Kubo S, Yokota H, Yokota H, Hayashi Y. The effect of lightcuring modes on the microleakage of cervical resin composite restorations. J Dent 2004; 32: 247 - 254.

2. Attar N, Korkmaz Y. Effect of two light-emitting diode (LED) and one halogen curing light on the microleakage of class V flowable composite restorations. J Contemp Dent Pract 2007; 2: 80 - 88.

3. Elhejazi AA. The effects of temperature and light Intensity on the polymerization shrinkage of light-cured composite filling materials. J Contemp Dent Pract 2006; 3: 12 $-21$.

4. Gumbau C. Fuentes lumínicas para la fotoactivación en odontología. [en línea] [fecha de acceso: agosto de 2008]. URL disponible en: http://www.blanqueamientodental. com/fuentes\%20luminicas.html

5. Microsoft Excel, 5.0 Microsoft Corporation, 1997

6. Stata corp, 2005. Stata Statistical Software: Release 9.0 Colleg Station, Tx: Stata Corporation L.P

7. República de Colombia. Ministerio de Salud. Resolución 008430 de 1993. [en línea] [fecha de acceso: agosto de 2008]. URL disponible en: www.urosario.edu.co/medicina/documentos/facultades/medicina/investigaciones/ resolucion_008430_1993.pdf

8. Radzi Z, Yahya NA, Zamzam N, Wood DJ. Light curing units: Tips for orthodontists. Annal Dent Univ Malaya [en línea] 2004 [fecha de acceso: julio de 2008] 11:13 - 23. URL disponible en: http://majlis.fsktm.um.edu.my/document.aspx?FileName $=364$.pdf

9. Blandón A, Tobón A, Valencia F, Jubiz O, Cuesta D. Intensidad de la luz de las lámparas de fotocurado de los consultorios odontológicos de los municipios de Envigado y Sabaneta 2002. [en línea]. [fecha de acceso: octubre de 2008]. URL Disponible en: http://www.acfo.edu.co/9 pub/xtras/2008_XIX_esp.pdf

10. Barghi N, Fischer D, Pham T. Revisiting the intensity output of curing lights in private dental offices. Compend Contin Educ Dent 2007; 28: 380 - 384.

11. Mitton BA, Wilson NH. The use and maintenance of visible light activating units in general practice. $\mathrm{Br}$ Dent $\mathrm{J}$ 2001; 191: 82 - 86 .

12. Säilynoja E, Shinya A, Koskinen M, Salonen J, Masuda T, Koide N, et al. Heat curing of UTMA-based hybrid resin: effects on the degree of conversion and cytotoxicity. Odontology; 2004; 92: 27 - 35.

13. Mowafy O, Badrawy W, Lewis D, Shokati B, Kermalli J, Soliman $\mathrm{O}$, et al. Intensity of quartztungsten-halogen light-curing units used in private practice in Toronto. $\mathrm{J}$ Am Dent Assoc 2005; 136: 766 - 773.

14. Guzmán H. Unidades de fotocurado. Revista de Operatoria Dental y Biomateriales [en línea]. [fecha de acceso: abril de 2008]. URL disponible en: http://www.encolombia.com/scodb3-unidades.htm

15. Keogh TP. Polimerización iniciada mediante luz: claros y oscuros de las nuevas técnicas. Ideas y Trabajos Odontoestomatológicos. 2001; 2: 29 - 37.

16. Manual del Operador de Demetron A.2. Kerr. 7 - 9.

17. Spectrum ${ }^{\circledR} 800$ lámpara de fotocurado lámpara de polimerización. [en línea]. [fecha de acceso: agosto de 2008]. URL disponible en: http://www.dentsply.es/DFU/esp/ Spectrum\%20800_esp.pdf

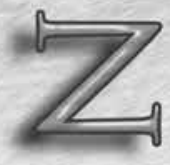

La Facultad de Odontología de la Universidad Santo

Tomás en concordancia con la visión institucional aspira a lograr:

Un compromiso efectivo de la comprensión renovada del hombre colombiano, su mundo y sus valores, en un contexto de salud integral para todos; el reconocimiento nacional e internacional, como comunidad académica, a través de la investigación, la producción intelectual y el intercambio académico; liderazgo en los procesos relacionados con la salud bucal de la comunidad, a nivel regional y nacional; la cualificación permanente de sus servicios a la comunidad para responder efectiva y oportunamente a sus usuarios; el fortalecimiento permanente de su accionar pedagógico mediante el desarrollo humano integral, la aplicación de tecnologías de punta y la proyección comunitaria. 